\title{
Triple Therapy in Canine Babesiosis - A Case Report
}

\author{
K.K. Ponnu Swamy*, T. Mohanapriya, P.A. Enbavelan, R.C. Sundararajan, \\ S. Saravanan and R. Ramprabhu
}

Department of Veterinary Medicine, Veterinary College and Research Institute, Tirunelveli627 358, Tamil Nadu Veterinary and Animal Sciences University, India

*Corresponding author

\section{A B S T R A C T}

Keywords

Canine Babesiosis,

Babesia gibsoni,

Triple therapy,

Clindamycin

Article Info

Accepted:

10 November 2019

Available Online:

10 December 2019
A male Dalmatian aged one year was presented with history of pyrexia, anorexia, lethargy and brownish urination. On clinical examination animal had high body temperature $\left(40.1^{\circ} \mathrm{C}\right)$, pale mucous membranes with petechiae and lymphadenopathy. Routine hematology showed anemia and examination of peripheral blood smear revealed the presence of piroplasms of Babesia gibsoni (+++). Triple therapy was initiated with Clindamycin @ $25 \mathrm{mg} / \mathrm{kg}$ PO q 12h, Metronidazole @ 15mg/kg PO q 12h and Doxycycline @ $5 \mathrm{mg} / \mathrm{kg}$ PO q $12 \mathrm{~h}$ for 10days. On the tenth day the animal recovered completely and peripheral blood smear was negative for the parasitic infection. The present study was carried out to evaluate the efficacy of triple therapy in natural infection of Babesia gibsoni in dog.

\section{Introduction}

Canine Babesiosis is one of the most important life threatening tick borne haemoprotozoan diseases of dogs caused by intraerythrocytic protozoan parasite of the genus Babesia reported worldwide. Babesia canis and Babesia gibsoni are the two organisms commonly known to infect the dogs are transmitted by ixodid tick vectors (Sunitha et al., 2011). B. canis, the larger organism (4 to $5 \mu \mathrm{m}$ ) is more common in the United
States. B. gibsoni the smaller one $(1-5 \mu \mathrm{m})$ has been recognized as an important pathogen that affects dogs in the Middle East, Africa, Asia, Europe, and many areas of the United States (Taboada and Merchant, 1991). A wide variation of clinical signs like anorexia, lethargy, haemolytic anaemia, icterus, vomiting and marked loss of body condition was reported in canine Babesiosis (Ettinger and Feldman 2005) along with clinicopathologic abnormalities like ascites, haemoglobinuria, hypoglycaemia, acid-base 
disturbances, azotemia and elevation in the levels of liver enzymes (Irwin, 2010).

A few drugs and drug combinations are used in the treatment of Canine Babesiosis often without complete parasite elimination and the dog usually become chronic carriers or present with recurrent episodes of acute infection (Baneth, 2018). Several drugs and drug combinations have been reported to be effective against Canine Babesiosis (Beugnet and Moreau, 2015).

\section{Case history and clinical observation}

A male one year old Dalmatian dog was presented to the Veterinary Clinical Complex with the history of pyrexia, anorexia, lethargy and brownish urination. On clinical examination the animal had high temperature, $\left(40.1^{\circ} \mathrm{C}\right)$, pale mucous membrane with petechiae and lymphadenopathy. The whole blood was collected in EDTA and clot activator for routine haematology and serum biochemical analysis. A thin peripheral blood smear was prepared and stained with Leishman- Giemsa stain. The peripheral blood smear revealed the presence of annular and signet ring shaped (Figure 1) piroplasms measuring 1.2 to $2 \mu \mathrm{m}$ in diameter in red blood cells indicating that the animal is infected with Babesia gibsoni (+++). The blood picture showed anaemic changes like anisocytosis, poikilocytosis, polychromasia, nucleated RBCS and neutrophilic leucocytosis with left shift due to marked systemic inflammatory response (Table 1). The case was diagnosed as Canine Babesiosis due to Babesia gibsoni infection. The serum biochemical analysis was inconclusive.

\section{Treatment and Discussion}

The typical clinical sign observed in Canine Babesiosis is haemolytic anaemia as parasitemia results in increased osmotic fragility of erythrocytes and serum haemolytic factors causing haemolysis (Jacobson and Clark, 1994). Clinical signs of canine babesiosis include fever, anorexia, depression, oliguria, haemoglobinuria, vomiting, lethargy, dehydration, icterus, pale mucous membranes, splenomegaly and dyspnoea (Irwin, 2010) which coincides with our case. The dog was treated with Oxytetracycline @ 20mg/Kg body weight intravenously, Imidocarb dipropionate @ $5.5 \quad \mathrm{mg} / \mathrm{kg}$ given intramuscularly and then repeated after two weeks and Diminazene aceturate @ 3.5 $\mathrm{mg} / \mathrm{Kg}$ intramuscularly once without success. On examination of peripheral blood smear the dog after treatment remained positive for $B$. gibsoni infection. Hence a combination therapy with Clindamycin @ 25mg/kg PO q 12h, Metronidazole @ 15mg/kg PO q 12h and Doxycycline @ 5mg/kg PO q 12h for 10 days was followed. This combination boosts the innate immunity and is known as the Marshall Protocol (Nandhini et al., 2016). The dog recovered uneventfully and the peripheral blood smear was negative for the parasitic infection after ten days.

The first treatment that has been shown to be effective against $B$. gibsoni is a combination of Atovaquone and Azithromycin. Unfortunately Atovaquone is not available in India and it's expensive for import. Moreover Possible Emergence of Drug-Resistant Variants of B. Gibsoni in clinical cases treated with Atovaquone and Azithromycin has also been reported (Wulansari et al., 2003). In a study the combination of the DoxycyclineEnrofloxacin-Metronidazole was found to be $83.3 \%$ effective in the treatment of Canine Babesiosis (Lin and Huang, 2010). Thus, it is concluded that the triple therapy with three antibiotics Clindamycin (CLDM), Metronidazole (MNZ), and Doxycycline (DOXY) as an efficacious alternative treatment strategy for $B$. gibsoni infection with no adverse effects. 
Table.1 Routine haematology

\begin{tabular}{|c|c|}
\hline Parameters & Observed Values \\
\hline Haemoglobin $(\mathbf{g} / \mathbf{d l})$ & 10.1 \\
\hline PCV(\%) & 23.8 \\
\hline $\mathbf{6}$ & 5.39 \\
\hline RBC $(\times \mathbf{1 0} / \boldsymbol{\mu l})$ & 34800 \\
\hline WBC $(\times \mathbf{3}$ / $\mathbf{\mu l})$ & 134900 \\
\hline Platelets $(/ \boldsymbol{\mu l})$ & 86 \\
\hline Neutrophils \% & 7 \\
\hline Lymphocytes \% & 4 \\
\hline Monocytes \% & 3 \\
\hline Eosinophils \% & \\
\hline
\end{tabular}

Fig.1 Blood smear (100 X, Leishman- Giemsa) Babesia gibsoni - Annular or signet ring shaped piroplasms

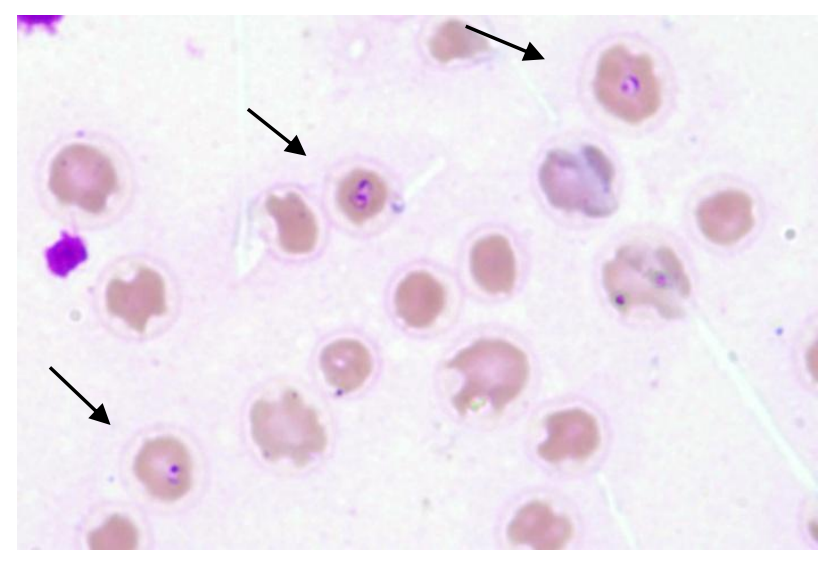

\section{References}

Beugnet, F., Moreau, Y., 2015. Babesiosis. Rev. Sci. Tech. 34, 627-639.

Baneth G. 2018. Antiprotozoal treatment of canine babesiosis. Vet Parasitol. 254:58-63

Ettinger SJ, Feldman EC. Text book of veterinary internal medicine. 6 . Missouri: W.B. Saunders Company; 2005. pp. 643-644

Irwin, P.J. 2010. Canine babesiosis. Vet. Clin. North Am. Small Anim. Pract. 40: 1141-1156.

Jacobson, L.S. and I.A. Clark, 1994. The pathophysiology of canine babesiosis:
New approaches to an old puzzle. $J$. $S$. Afr. Vet. Assoc., 65: 134-145.

Lin M-Y, Huang H-P (2010). Use of doxycycline-enrofloxacinmetronidazole combination with and without injections of diminazene diaceturate to treat naturally occurring canine babesiosis caused by Babesia gibsoni. Acta Vet Scand 52:27

Nandini MK, Poonam Vishwakarma and Ansar Kamran. 2016. New therapeutic protocol for canine babesiosis: a case report. J Dairy Vet Anim Res. 3(3):112-113

Sunitha Karunakaran, U. N. Pillai, H. P. Sasidharan. 2011. Babesia gibsoni 
infection in a German Shepherd dog. Vet. World. 4(6): 269-270.

Schoeman J P. 2009. Canine babesiosis. Onderstepoort J Vet Res. 76(1): 59-66.

Taboada J, Merchant SR: Babesiosis of companion animals and man. Vet Clin North Am Small Anim Pract. 21:103-
123, 1991.

Wulansari R, Wijaya A, Ano H, et al., 2003. Lymphocyte subset and specific IgG antibody levels in Clindamycin treated and untreated dogs experimentally infected with Babesia gibsoni. J Vet Med Sci. 65(5):579-584.

\section{How to cite this article:}

Ponnu Swamy, K. K., T. Mohanapriya, P. A. Enbavelan, R. C. Sundararajan, S. Saravanan and Ramprabhu, R. 2019. Triple Therapy in Canine Babesiosis - A Case Report. Int.J.Curr.Microbiol.App.Sci. 8(12): 964-967. doi: https://doi.org/10.20546/ijcmas.2019.812.124 